\title{
INTELLIGENT WIRELESS ULTRASONIC DEVICE FOR DAMAGE DETECTION OF METALLIC STRUCTURES
}

\author{
Manish Man Shrestha*,**, Bibek Ropakheti*, Uddhav Bhattarai*, Ajay Adhikari* and Shreeram \\ Thakur* \\ *Cosmos College of Management and Technology, Lalitpur, Nepal. \\ ${ }^{* *}$ CTO, Samadhan Engineering Pvt. Ltd., Lalitpur, Nepal.
}

\begin{abstract}
In today's world, it is necessary to monitor structures for possible damages. A failure to monitor the structures properly can cause structural catastrophe. Many researchers have worked on the low-power ultrasonic device to monitor the structures. In this research, we present an intelligent ultrasonic device (IUD) to monitor and detect the damages on the structures. The device uses microcontroller, actuator interface circuit, sensor interface circuit and radio frequency (RF) modem. The microcontroller has in-built high-speed analog-to-digital converter (ADC), digital-to-analog converter (DAC) and floating-point unit for signal processing. The controller generates the tone-burst signal and send it to actuator interface circuit. The actuator interface circuit conditions the received signal from the microcontroller and drives the Piezoelectric Transducer (PZT) actuator. The actuator generates an ultrasonic wave in the structure. The wave is then sensed by PZT sensors. The sensor interface circuit selects the signal from desired PZT sensor and send it to the microcontroller for further processing. The microcontroller digitizes the signal and computes the damage index and only if the damage is severe, it will send data wirelessly to the nearby PC. To test the device, iron specimen was prepared, PZT actuator and PZT sensor was mounted on it. The artificial crack was then induced on the specimen. The ultrasonic wave was then collected from the structure. By analyzing the ultrasonic wave, the device successfully detected the induced crack in the structure. The future work will be to use GSM modem so that the device can be monitored in the real time from the remote location.
\end{abstract}

Keywords: Structure health monitoring; Tone-burst generator; Intelligent ultrasonic device; Non-destructive evaluation.

\section{INTRODUCTION}

The metallic structures can adulterate with time due to ageing, fatigues, external loading and environmental influences. If structure is not monitored carefully, it can fail and may lead to serious incidents. To prevent such failures, it is necessary to provide highest level of quality and safety measures. In recent years, many researchers are doing research to utilize the wireless communication with ultrasonic wave for structure health monitoring of metallic structures. However, majority of the current devices are not intelligent and also have low data throughput with low amplitude of actuation signal. With this research, we present wireless intelligent ultrasonic device, which detects damage locally through wireless communication. The generates high amplitude actuation signal and uses Piezoelectric Transducer (PZT) sensor and actuator for precise measurement of the damages in the structure. The device can also compute the damage index of the structure in real time and only alert the Personal Computer (PC) if damage is severe.

The ultrasonic guided wave is intensively used to estimate damage in the structures ${ }^{1}$. Lissenden et al. has successfully

Author for Correspondence: Manish Man Shrestha, Cosmos College of Management and Technology, Lalitpur, Nepal and CTO, Samadhan Engineering Pvt. Ltd., Lalitpur, Nepal.

Email: mshrestha789@gmail.com

Received: 6 Sep 2020; Review: 18 Dec 2020; Accepted: 5 Jan 2020.

Doi: https://doi.org/10.3126/sw.v14i14.34979 
use the guided wave to localize the damage in the structure. The authors have used the array of sensors to localize the damage. Giurgiutiu ${ }^{2}$ has reviewed the different methods to detect the damage using ultrasonic wave. The paper shows the reliability and limitation of the method. Although this traditional wired system can detect damage convincingly, installation of wired system for active sensing can be expensive and may take several months for installation. On the other hand, wireless system can be cheap and cost effective.

Heo et al. ${ }^{3}$ have developed a smart monitoring system based on ubiquitous computing technique. The system uses Transmission Control Protocol/Internet Protocol (TCP/IP) network protocol over a Bluetooth technology. The use of Bluetooth technology, however, limits its data throughput. Lynch J. P. et al. ${ }^{4,5}$ have developed the low power wireless monitoring system and successfully detect the damage in the structure but the device is not intelligent and since the device doesn't use any standard communication protocol it is not directly compatible with other wireless devices.

Pertsch et al. ${ }^{6}$ have utilized the modern embedded and wireless technology to develop the intelligent wireless device for continuous monitoring of the structure. The research clearly shows the reliability of the modern technology to detect the damage. Nevertheless, the device doesn't compute damage index in real time and doesn't communicate with standard communication protocol.

In this paper, we explored the available modern technologies and the impacts it can make in the field of structural health monitoring. We found out the option of computing damage index in the real time with lesser human intervention.

\section{OVERVIEW}

Fig. 1 shows the overview of the intelligent ultrasonic device (IUD) system. The system consists of two main parts: IUD and ground PC. The IUD is placed on the desired metallic structure. The sensor and actuator are attached to it. The IUD first generates the tone-burst signal of amplitude $15 \mathrm{Vp}$-p, 6 cycle and $138 \mathrm{KHz}$ frequency. It then actuates the PZT actuator. The PZT actuator in-turn generates the ultrasonic wave in the structure. The wave travel through the structure and reaches the PZT sensor. The sensor on receiving the wave, converts it into electrical signal and send it back to the IUD. IUD on receiving the signal, condition and digitize the received signal. The digital signal is then processed by its controller to see whether there is a damage in the structure or not. If the device detects the damage, the damage index is sent to the PC via wireless communication. The PC can even request a raw data from the IUD for further analysis.

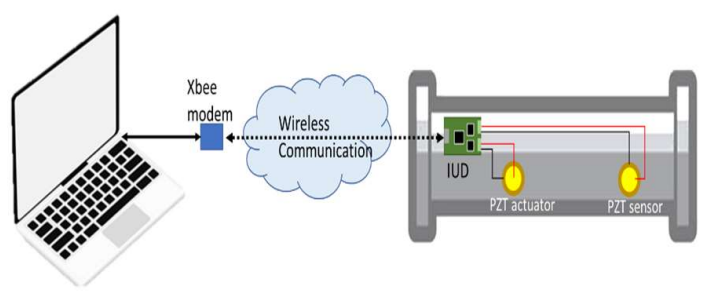

\section{Figure 1: Overview of wireless IUD system}

\section{DEVELOPMENT OF IUD SYSTEM}

Fig. 2 shows the development concept of IUD. The IUD board has actuator interface circuit, sensing interface circuit, microcontroller and wireless modem. Table 1 shows the specification of IUD.

Table 1. IUD specification

\begin{tabular}{|l|l|}
\hline Specification & Performance \\
\hline Power supply & $12 \mathrm{~V}$ \\
\hline Microcontroller & $\begin{array}{l}168 \mathrm{MHz}, \mathrm{ADC}: 10 \mathrm{bit}, 2.5 \\
\text { MSPS, FPU }\end{array}$ \\
\hline Actuator signal & $138 \mathrm{kHz}, 15 \mathrm{Vp}-\mathrm{p}, 6$ cycle \\
\hline Communication & Wireless \\
\hline
\end{tabular}

\section{Actuator interface circuit}

Actuator interface circuit is designed so that the IUD can drive PZT with necessary amplitude and frequency. To drive the PZT, the actuation signal must be of sufficient amplitude and frequency. The design mainly consists of two parts: microcontroller and the circuit. The microcontroller has in-built DAC, which is used to generate analog signal pattern to drive the actuator. As the actuation signal is a sine wave with increasing and then decreasing amplitude, eq. (1) is developed. Using the equation, the firmware is written 
and signal is successfully generated. To increase the frequency of the signal, DMA controller is used.

lamb wave
$=\left\{\begin{array}{l}e^{\frac{t 12}{2}} \cdot \cos (n \cdot t 1) \quad \text { for } t 1<a \\ {\left[e^{\frac{t 2}{2}} \cdot \cos (n \cdot t 1)\right]^{-1} \quad \text { for } t 1 \geq a \wedge t 1<t}\end{array}\right.$
The amplitude of the signal is of low amplitude and could drive PZT actuator properly, so the actuator circuit is developed. Fig. 2 shows the schematic of actuator circuit. The circuit consists of buffer amplifier, gain amplifier with compensation capacitor and resistor to drive PZT load and push-pull amplifier to increase the final amplitude.

(Eq. 1)

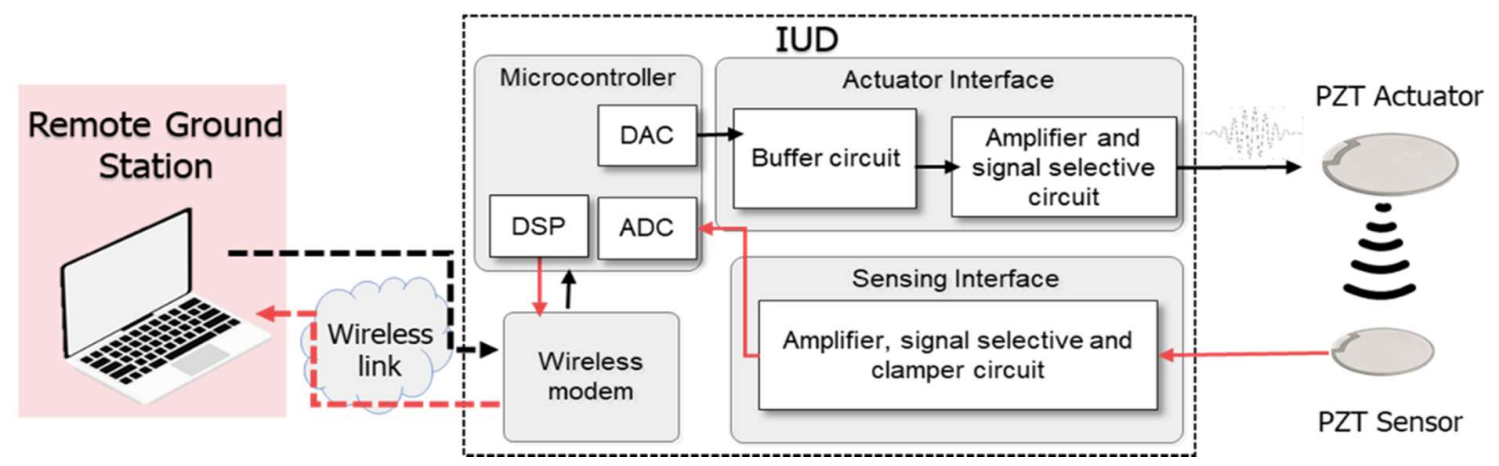

Figure 2: Block Diagram of IUD system
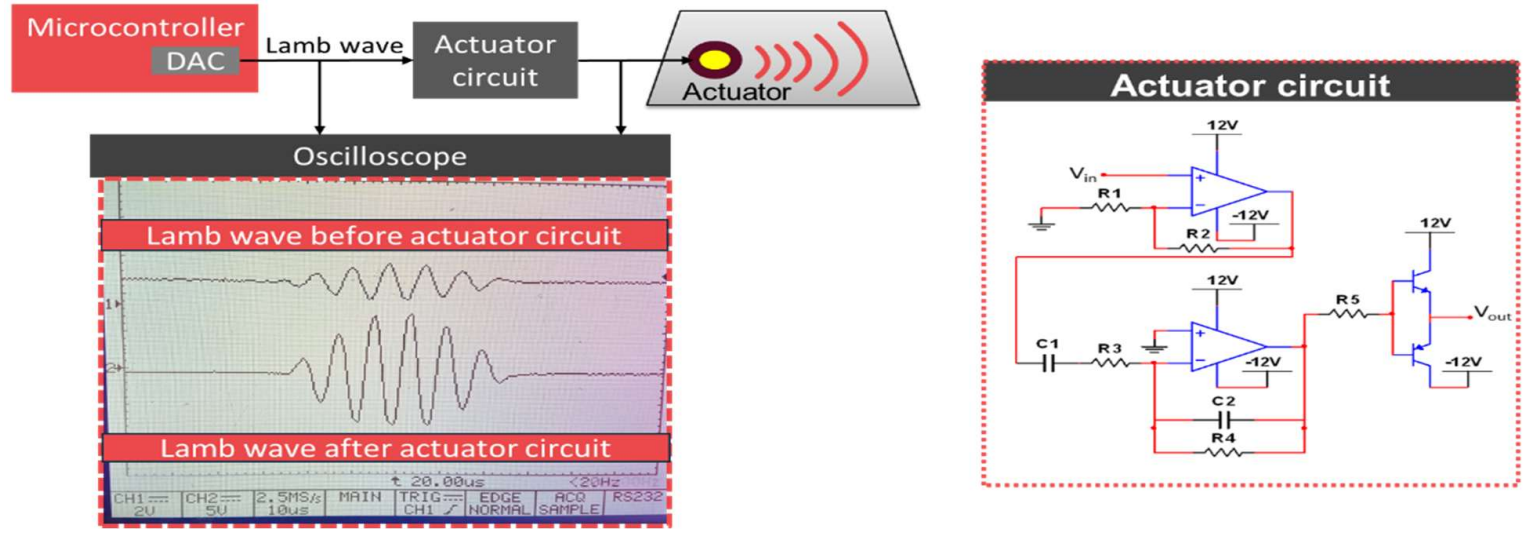

Figure 3: Actuator circuit and actuator circuit testing

By using the circuit, the lamb wave of amplitude $15 \mathrm{Vp}-\mathrm{p}$, 6 cycle and $138 \mathrm{kHz}$ frequency is successfully generated.

\section{Sensing interface circuit}

Sensor interface circuit is designed to process the ultrasonic wave from the PZT sensor. The microcontroller uses this wave to calculate damage index. However, the wave from the sensor has very-low amplitude and has both positive and negative voltage. To make the signal suitable to process, the amplitude should be increased and voltage level should be above zero. The sensor interface circuit consists of charge amplifier, voltage level shifter, clipper circuit and ADC. The gain of the amplifier is set to 100, voltage level shifter shifts the negative voltage to positive level, clipper circuit makes sure that the voltage doesn't cross upper limit of $\mathrm{ADC}$ and $\mathrm{ADC}$ digitized the analog data. The circuit uses the in-built ADC of the microcontroller. The sampling rate of the ADC is set to 2.5 million samples per second (MSPS). To get this sampling rate, DMA controller of the microcontroller is used. As PZT sensor stores charge in accordance to the ultrasonic wave, the charge amplifier with high input impedance is designed.

Fig. 4 shows that the sensor interfacing circuit. The circuit has successfully detected the ultrasonic wave from the sensor. The oscilloscope shows the detected ultrasonic wave, captured through the sensor interface circuit. 


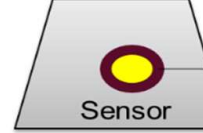

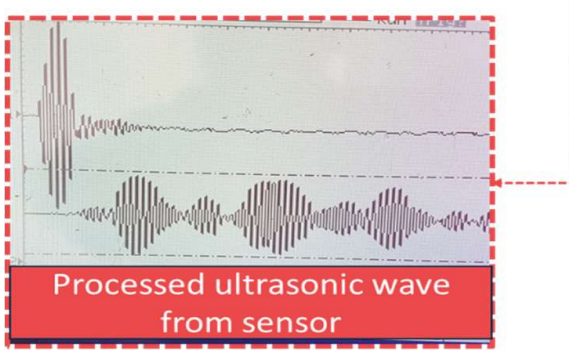

Figure 4: Sensor circuit and Sensor circuit testing

\section{Digital signal processing}

The damage index is calculated in the microcontroller. The microcontroller is chosen with the in-built floating-point unit (FPU) so that digitized signal can be processed more precisely. The digitized ultrasonic wave is first filtered with low pass filter with cut-off frequency of $300 \mathrm{kHz}$. The $6^{\text {th }}$ order Butterworth filter is designed for this purpose. The signal is then further processed to calculate damage index. Time domain - root mean square (TD-RMS) algorithm is used in the controller to calculate the damage index. TDRMS algorithm is shown in the eq. (2).

$$
D I_{T D R M S}=\frac{\int_{t_{1}}^{t_{2}}\left|S_{m}(t)-S_{b}(t)\right|^{2} d t}{\int_{t_{1}}^{t_{2}}\left|S_{b}(t)\right|^{2} d t}
$$

\section{Wireless communication}

The IUD uses wireless communication to communicate with the PC. The modem used for wireless communication is $\mathrm{X}$ Bee modem. The $\mathrm{X}$ Bee modem uses a ZigBee protocol. The ZigBee is IEEE 802.15.4 standard, low

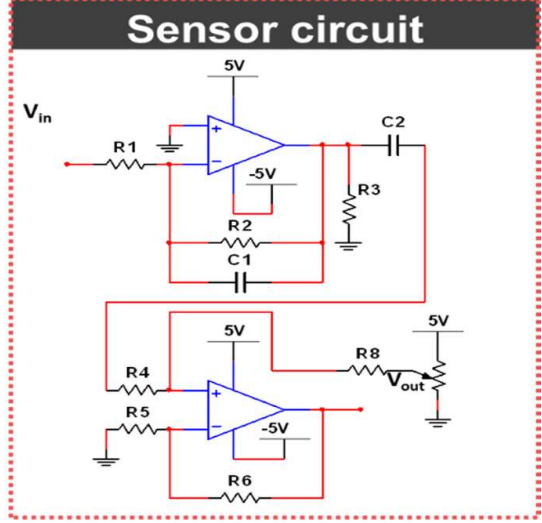

power, low data transmitting wireless protocol. The architecture of ZigBee includes three parts namely coordinator, router and end device. The coordinator is the source of the ZigBee networks and manages information and security across the network. A router acts as intercessor device for two-way communication between router and other devices. It has four layers: 1) physical layer, 2) MAC layer, 3) network layer and 4) application layer.

To initialize communication, first both $\mathrm{x}$ bee modems of transmitter and receiver is set up with same channel name, ID and baud rate (9600). The command "CE (Coordinator Enable)" of transmitter is set to "coordinator" and CE of receiver is set to "End point". After this command, the modems are joined to IUD and PC. The communication is done using (Universal Asynchronous Receiver/ Transmitter) UART.

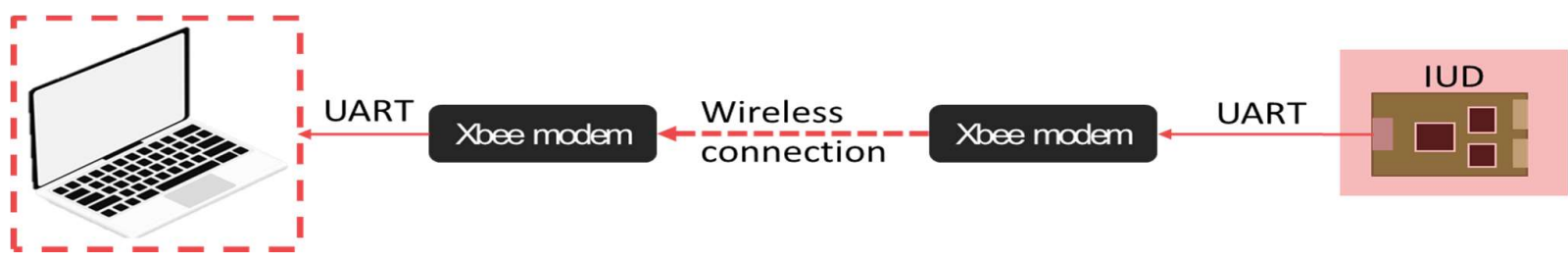

Figure 5: Overview of wireless communication of IUD system

\section{Firmware development}

The firmware is developed on open source real time operating system (RTOS) platform called "free RTOS". The flowchart of the firmware is given below 
Initializes microcontroller, RTOS, and its peripherals such as ADC, DAC and UART for wireless communication

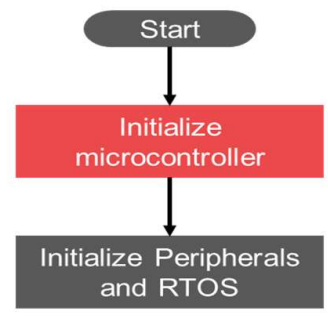

Figure 6: Flowchart of IUD firmware

\section{TESTING OF IUD}

To evaluate the performance of IUD regarding damage detection, iron specimen is chosen. The PZT actuator and sensor is mounted on the structure as shown in fig. 7 . The

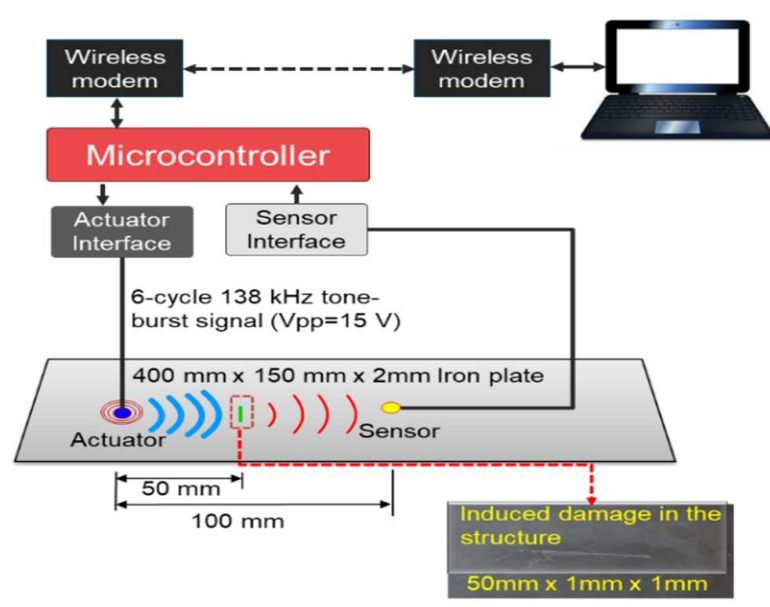

ultrasonic wave is first collected in intact condition of the specimen. Then the artificial crack is induced in it. The ultrasonic wave is again collected from the specimen. The wave from both conditions is compared and damage index is calculated
Filter data from sensor to remove noises, implements damage index algorithm, and if damage is severe, send damage index

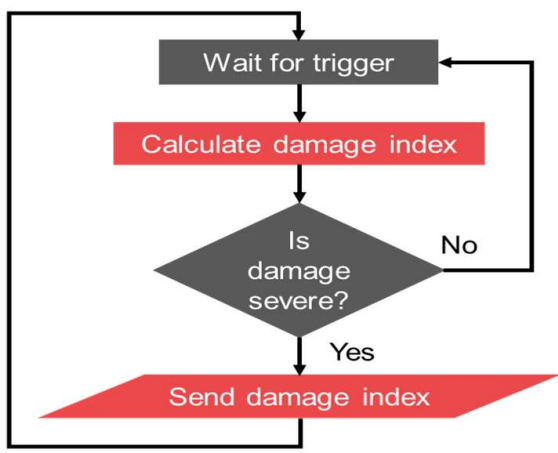

Figure 7: IUD test setup

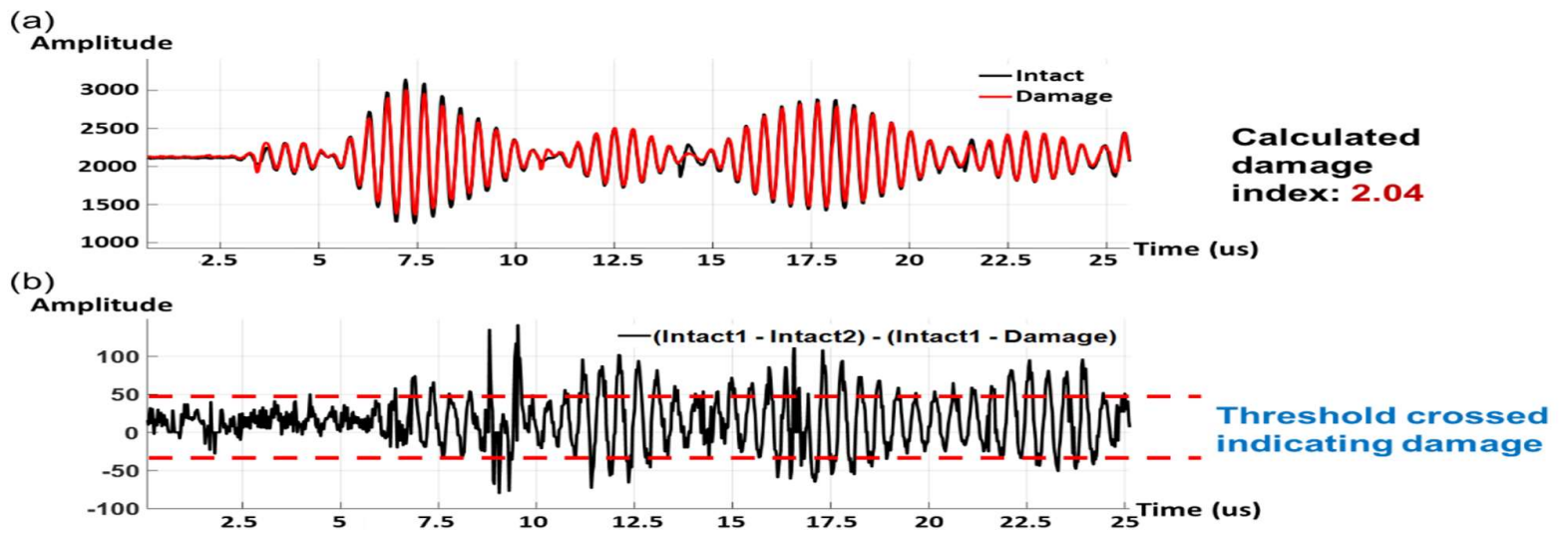

Figure 8: Comparison of the signal before and after damage on the iron plate (a) amplitude comparison (b) amplitude difference 
From fig. 8, it is clear that the damage has been successfully detected with damage index of 2.04 .

\section{CONCLUSION}

From this research, we conclude that, it is possible to build an intelligent device that can detect damage by itself without any human intervention. The modern controllers are powerful enough to calculate the damage index by itself. The wireless communication is also reliable to send the damage index to the PC. With careful design and calibration, it is possible to both actuate and sense the PZT sensors. Furthermore, PZT sensors are reliable to generate and detect the ultrasonic wave in the structure. With the use of high precision components, the obtained results are found to be highly correlated with the actual damages in the structure.

\section{ACKNOWLEDGEMENT}

This project is jointly funded by Pokhara University Research Management Cell (PURC), RMC-Cosmos College of Management and Technology and Samadhan Engineering under the research topic "Development of low power multi-channel intelligent wireless device for health monitoring of metallic struct

\section{REFERENCES}

1. Cliff J. Lissenden and Joseph L. Rose. 2008. Structural health monitoring of composite laminates through ultrasonic guided wave beam forming. Engineering Science and Mechanics, The Pennsylvania State University, University Park, PA.

2. Victor Giurgiutiu. 2008. Structural health monitoring with piezoelectric wafer active sensors. New York: Elsevier Academic Press.1-3. G.

3. Gwanghee Heo and Joonryong Jeon. 2010. A smart monitoring system based on ubiquitous computing technique for infra-structural system: centering on identification of dynamic characteristics of selfanchored suspensionbridge. KSCE J. Civ. Eng. 13: 333-337.

4. Jerome P. Lynch, Yang Wang, Kenneth J. Loh, Jin-Hak Yi and Chung-Bang Yun, 2006, Performance monitoring of the Geumdang bridge using a dense network of high-resolution wireless sensors Smart Mater. Smart Materials and Structures. 15:1561-1575.

5. Jerome Peter Lynch, 2005, Design of a wireless active sensing unit for localized structural health monitoring. Structural Control Health Monitoring. 12:405-23

6. Alexander Pertsch, Jin-Yeon Kim, Yang Wang and Laurence Jacobs, 2010, An intelligent stand-alone ultrasonic device for monitoring local structural amage: implementation and preliminary experiments. Smart materials and structures. 20 (1). 